\title{
Bekanamycin Sulfate
}

National Cancer Institute

\section{Source}

National Cancer Institute. Bekanamycin Sulfate. NCI Thesaurus. Code C76153.

The sulfate salt form of kanamycin B, a minor component of the kanamycin complex, an aminoglycoside antibiotic isolated from Streptomyces kanamyceticus, with antibacterial activity. 